\title{
Visuotopic Cortical Connectivity Underlying Attention Revealed with White-Matter Tractography
}

\author{
Adam S. Greenberg, ${ }^{1,2}$ Timothy Verstynen, ${ }^{2,3,4}$ Yu-Chin Chiu, ${ }^{6}$ Steven Yantis, ${ }^{7}$ Walter Schneider, $, 2,3,4,5$ \\ and Marlene Behrmann ${ }^{1,2}$ \\ ${ }^{1}$ Department of Psychology, Carnegie Mellon University, ${ }^{2}$ Center for the Neural Basis of Cognition, Carnegie Mellon University and University of Pittsburgh, \\ ${ }^{3}$ Learning Research and Development Center and ${ }^{4}$ Department of Psychology, University of Pittsburgh, ${ }^{5}$ Department of Neurosurgery, University of \\ Pittsburgh Medical Center, Pittsburgh, Pennsylvania 15213, ${ }^{6}$ Department of Psychology, University of California, San Diego, La Jolla, California 92093, and \\ ${ }^{7}$ Department of Psychological and Brain Sciences, The Johns Hopkins University, Baltimore, Maryland 21218
}

Visual attention selects behaviorally relevant information for detailed processing by resolving competition for representation among stimuli in retinotopically organized visual cortex. The signals that control this attentional biasing are thought to arise in a frontoparietal network of several brain regions, including posterior parietal cortex. Recent studies have revealed a topographic organization in the intraparietal sulcus (IPS) that mirrors the retinotopic organization in visual cortex, suggesting that connectivity between these regions might provide the mechanism by which attention acts on early cortical representations. Using white-matter imaging and functional MRI, we examined the connectivity between two topographic regions of IPS and six retinotopically defined areas in visual cortex. We observed a strong positive correlation between attention modulations in visual cortex and connectivity of posterior IPS, suggesting that these white-matter connections mediate the attention signals that resolve competition among stimuli for representation in visual cortex. Furthermore, we found that connectivity between IPS and V1 consistently respects visuotopic boundaries, whereas connections to V2 and $\mathrm{V} 3 / \mathrm{VP}$ disperse by $60 \%$. This pattern is consistent with changes in receptive field size across regions and suggests that a primary role of posterior IPS is to code spatially specific visual information. In summary, we have identified white-matter pathways that are ideally suited to carry attentional biasing signals in visuotopic coordinates from parietal control regions to sensory regions in humans. These results provide critical evidence for the biased competition theory of attention and specify neurobiological constraints on the functional brain organization of visual attention.

\section{Introduction}

Visual attention is the process by which behaviorally relevant visual information is selected and irrelevant information is ignored. The performance advantage observed when attention is deployed to a spatial location before stimulus onset (Bashinski and Bacharach, 1980; Posner et al., 1980; Egeth and Yantis, 1997) relies on a number of still poorly understood neural mechanisms. Neuroimaging and neurophysiological evidence supports a biased competition model (Desimone and Duncan, 1995) of visual attention in which stimuli compete for neural representation in retinotopically organized visual cortex. This competition is resolved by directing attention to the target, resulting in enhanced neural activity to attended stimuli and, simultaneously, decreased activity in neurons representing distracters. Although this competition plays out in visual cortex (Kastner et al., 1998; De Weerd et al., 1999; Reynolds et al., 1999; Kastner and Ungerleider, 2001), the source of signals that control this attentional

Received 0ct. 26, 2011; revised Jan. 3, 2012; accepted Jan. 5, 2012.

Author contributions: A.S.G., Y.-C.C., S.Y., and M.B. designed research; A.S.G. and T.V. performed research;A.S.G., T.V., and W.S. contributed unpublished reagents/analytic tools; A.S.G. analyzed data; A.S.G. wrote the paper.

This work was supported by NIH Grant R01-MH54246 (M.B.). We thank Adrian Nestor and Jeffrey Phillips for helpful discussions about analyses.

Correspondence should be addressed to Dr. Adam S. Greenberg, Department of Psychology, Carnegie Mellon University, Baker Hall 342-C, 5000 Forbes Avenue, Pittsburgh, PA 15213. E-mail: agreenb@cmu.edu.

DOI:10.1523/JNEUROSCI.5419-11.2012

Copyright $\odot 2012$ the authors $\quad 0270-6474 / 12 / 322773-10 \$ 15.00 / 0$ biasing lie in a network of frontoparietal regions (Corbetta, 1998; Kastner and Ungerleider, 2000).

A critical unanswered question is how signals from the frontoparietal network modulate the competition for selection in visual cortex. While visual cortex retinotopy depends upon stimulus location on the retina (Holmes, 1918; Wandell et al., 2007), the recently discovered topographic organization of the intraparietal sulcus (IPS) (Sereno et al., 2001; Silver et al., 2005; Swisher et al., 2007; Saygin and Sereno, 2008) is dependent upon the location to which attention is deployed (Silver and Kastner, 2009). Importantly, attention topography and stimulus-based retinotopy overlap precisely in visual cortex (Tootell et al., 1998). This overlap suggests a straightforward hypothesis: that direct structural connectivity between the two topographic maps might provide the mechanism by which attentional control signals bias the processing of spatially represented visual stimuli.

Only indirect evidence of this topographic connectivity hypothesis has been observed (Lauritzen et al., 2009; Uddin et al., 2010). Here, we explore the structural connectivity between corresponding topographic regions of IPS and visual cortex, examining whether the connectivity pattern respects the visuotopic organization and the degree to which these white-matter connections underlie attentional control of visual representations.

We used a combination of high angular resolution diffusion spectrum imaging (DSI) (Wedeen et al., 2005), reconstruction, 
and deterministic tractography (Verstynen et al., 2011). This approach was recently used to map detailed somatotopy in the cortocospinal pathway (Verstynen et al., 2011), organized at a spatial scale similar to that of topographic maps in the visual system. This technique allowed us to perform the fine-grained analyses necessary to examine the visuotopic connectivity between brain regions.

In tandem with DSI, we used fMRI to generate seed regions for fiber tractography. Corresponding visual field locations were mapped using stimulus-evoked methods (visual cortex) and attention-evoked methods (IPS). We examined the connectivity between two topographic regions of IPS along with six retinotopically defined areas in visual cortex. Furthermore, we measured attention modulations in each region and correlated these with measures of structural connectedness. Finally, we tested the degree of topographic consistency in fiber streamlines connecting IPS and visual cortex.

\section{Materials and Methods}

Subjects. Subjects were five healthy individuals (mean age, 28 years; range, 22-32; four males) recruited from the Pittsburgh university community. All subjects provided informed written consent in compliance with the institutional review boards of both Carnegie Mellon University and the University of Pittsburgh.

Procedural details. All magnetic resonance images were acquired using a $3 \mathrm{~T}$ Siemens scanner at the University of Pittsburgh Medical Center. DSI data were acquired during a single $1 \mathrm{~h}$ scanning session. fMRI data were acquired over two additional sessions of $2 \mathrm{~h}$ each. Stimuli in the three fMRI experiments were backprojected onto a screen mounted to the scanner bore rear. Stimuli were generated and displayed via MATLAB scripts (MathWorks) created with Psychophysics Toolbox extensions (Brainard, 1997; Pelli, 1997). Subjects viewed the screen (via a head coil-mounted mirror) at a distance of $68 \mathrm{~cm}$ and responded via a MR-compatible, fiber-optic button response glove worn on the dominant hand. No stimuli were present during the DSI session.

Brain activation: $f M R I$ stimuli. Each subject performed eight runs (four per session; $308 \mathrm{~s}$ each) of the retinotopic meridian-mapping experiment. Stimuli consisted of a bowtie-shaped wedge of contrast-reversing checkerboard at $8 \mathrm{~Hz}$ on a median gray background, encompassing $45^{\circ}$ of polar angle at maximum (see Fig. 1) (for details, see Slotnick and Yantis, 2003). Horizontal and vertical meridian stimulation alternated (18 s each), with eight epochs per run. A central fixation disk $\left(0.36^{\circ}\right.$ diameter $)$ was rendered in either black or white, changing color randomly every 5 , 10 , or $15 \mathrm{~s}$.

Subjects performed eight runs (four per session; $290 \mathrm{~s}$ each) of the stimulus-based location-mapping experiment. Here, a central fixation disk alternating between black and white (see above), and six rapid serial visual presentation (RSVP) (Potter and Levy, 1969; Reeves and Sperling, 1986) streams of white letters on a median gray background were presented in fixed-width Monaco font at $5^{\circ}$ eccentricity (see Fig. 2a). RSVP streams updated every $150 \mathrm{~ms}$, with no temporal gap between letters. Only one RSVP stream was present at any one time for $15 \mathrm{~s}$ before moving to the next location. Stimulus locations were presented in clockwise order, cycling through all six locations, three times per run.

Subjects performed 16 runs ( 8 per session; 290 s each) of the attentionmapping experiment. Stimuli were identical with that of the locationmapping experiment with two exceptions: (1) the fixation disk did not change color, it was rendered in white throughout, and (2) all six RSVP streams were present in the display at all times during each run. Subjects began each run by attending to the upper-right RSVP location of the display.

All three experiments contained epochs of fixation-only at the beginning and end of each run to allow for sampling of the baseline hemodynamic response.

Brain activation: fMRI analysis. All fMRI data were collected on an eight-channel, parallel imaging head coil. We acquired a high-resolution anatomical scan using a T1-weighted magnetization-prepared gradient echo pulse sequence (TR, $2300 \mathrm{~ms}$; TE, $2.98 \mathrm{~ms}$; flip angle, $9^{\circ}$; matrix, $256 \times 240 ; 160$ slices; voxel size, $1 \mathrm{~mm}^{3}$ ) during each fMRI session. From this dataset, we segmented gray from white matter and generated cortical surface representations for each hemisphere using FreeSurfer (Dale et al., 1999; Fischl et al., 1999).

Blood oxygenation level-dependent (BOLD) fMRI signal was measured using a T2*-weighted EPI sequence (TR, $2000 \mathrm{~ms}$; TE, $29 \mathrm{~ms}$; flip angle, $90^{\circ}$; matrix, $70 \times 70 ; 38$ slices; voxel size, $3 \mathrm{~mm}^{3}$ ). Functional data were analyzed with the AFNI/SUMA software package (Cox, 1996; Saad et al., 2004) and custom MATLAB scripts. Before analysis, all EPI runs from individual subjects were slice-time corrected, motion-corrected to an average EPI image volume from the first run acquired in each session, and converted to percentage signal change values normalized to the mean of each run. Data were then coregistered to the anatomical volume from which surfaces were generated. The EPI volumes from each run (within experiment) were then concatenated in time and mapped to the cortical surface representation using a method outlined previously (Greenberg et al., 2010). Briefly, time series were mapped to nodes on the smoothed gray/white boundary surface by taking the average of voxel data that lie between the gray/white and pial surfaces. No spatial smoothing was performed to preserve fine spatial correspondence between functional and anatomical data. Statistical analyses were then performed entirely on these surface-mapped data. A canonical hemodynamic response function (Cohen, 1997) was convolved with a model of the timing of the stimulation epochs during the experiment, yielding regressors corresponding to the conditions of interest in each of the three experiments. Fixation-only blocks were left as unmodeled time points to provide an estimate of the baseline variance in the model. Additional regressors accounting for variance due to linear and quadratic drifting as well as constant offset were included in the general linear model analysis (GLM) as nuisance regressors. Together, these were submitted to a multiple regression GLM (Friston et al., 1995).

In the meridian-mapping experiment, regressors for vertical and horizontal meridian conditions were contrasted and resulting functional maps were thresholded at a corrected familywise error rate of $p<0.05$. Borders between visual areas were then hand-drawn on the cortical surface by following the maximal path of activation, anteriorly from the occipital pole.

In the visual-mapping experiment, six regressors of interest corresponding to the six RSVP stream locations were contrasted. For the left hemisphere, each of the three locations in the right visual field (RVF) were contrasted against the other two locations, generating three functional maps, which were then thresholded at a corrected familywise $\alpha$ of 0.05 . Three corresponding functional maps were generated for the right hemisphere as well. Regions of interest (ROIs) were restricted to contiguous clusters within the visual area borders, defined previously by the meridian-mapping results. ROIs were hand-drawn around significant functional activations for each of the three locations per hemisphere. In $\mathrm{V} 1$, one ROI was drawn per mapped location. In V2 and V3/VP, two regions were identified in each of the dorsal and ventral regions identified in the meridian-mapping experiment. Because the horizontal meridian is represented in both dorsal and ventral portions of V2 and V3/VP, we identified ROIs for the middle RVF and LVF location in each. As expected, for dorsal V2 and V3/VP, we only observed activations for the lower visual field locations; and in ventral V2 and V3/VP, we only observed activations for the upper visual field locations. ROIs were drawn around each of these functionally defined regions resulting in five middle location ROIs (one in each of $\mathrm{V} 1, \mathrm{~V} 2 \mathrm{~d}, \mathrm{~V} 2 \mathrm{v}, \mathrm{V} 3 \mathrm{~d}$, and $\mathrm{VPv}$ ), three upper ROIs (V1v, V2v, VPv), and three lower ROIs (V1d, V2d, V3d), in each hemisphere.

In the attention-mapping experiment, six regressors of interest were generated similarly to the location-mapping experiment, except that epochs were restricted to times when subjects attended to each location. Functional maps were generated and thresholded at a corrected familywise $\alpha$ of 0.05 . ROIs in IPS were generated using the same contrast, thresholding, and contiguity procedures as those in visual cortex. However, because the most posterior end of IPS encroaches on retinotopic occipital cortex, IPS ROIs were restricted to regions outside (anterior to) the area significantly activated by meridian mapping, to avoid retino- 
topic cortex (and, thus, IPS-0). Within the anatomical confines of the IPS and its immediately surrounding gyri in each hemisphere, we searched for restricted regions of cortex that varied from upper to middle to lower contralateral visual field attention activation (see Fig. $2 b$ ). Functionally defined ROIs were drawn at these three locations in each of the first two such visuotopic maps identified, moving anteriorly from the posteriormost region of IPS (but still anterior to retinotopically organized cortex). The IPS "map ROIs" [that is, those that defined all of posterior IPS (pIPS) or anterior IPS (aIPS)] were hand-drawn to include only the smaller, attention-mapped IPS ROIs and the cortical space between them on the surface representation.

For the attention BOLD signal analysis, we performed an additional GLM in which all three contralateral visual field regressors were pooled, yielding the average BOLD signal change during all epochs of attention to the contralateral visual field. This signal was then spatially averaged within each map ROI and submitted to a two-way ANOVA with three levels of visual area (V1, V2, V3/VP) and two levels of $z$-plane position (dorsal, ventral).

Structural connectivity: DSI. Our approach (Verstynen et al., 2011) combines high angular resolution DSI (Wedeen et al., 2005) with highdirection reconstruction of the orientation distribution function (ODF) and deterministic tractrography. This method affords increased accuracy over conventional diffusion MR imaging (such as diffusion tensor imaging) primarily due to its superior resolution of fiber crossings (Hagmann et al., 2006), subvoxel seeding, and interpolation of diffusion data during the deterministic tractography process (Yeh et al., 2010). Recently, this approach was used to map detailed somatotopy in the corticospinal pathway (Verstynen et al., 2011), which is organized at a similar scale to the visuotopic maps we identified in the current study. DSI data were collected on a 32-channel head coil using a $43 \mathrm{~min}, 257$ direction scan. We used a $2.4 \mathrm{~mm}$ isotropic, twice-refocused spin-echo EPI pulse sequence with multiple $q$ values (TR, $9916 \mathrm{~ms}$; TE, $157 \mathrm{~ms} ; b$-max $=7000 \mathrm{~s} / \mathrm{mm}^{2}$ ). All subjects were experienced at remaining still, and extra padding around the head was provided to minimize motion during the scan. Before reconstruction, we performed a quality check on the DSI data. Scans with low signal-to-noise from movement (determined as the $\sigma / \mu$, variance/mean, of the diffusion signal across all volumes in the scan) were reacquired on subsequent dates and the best scanning session was used for analysis. Also, no corrections for eddy currents or geometric distortion were performed due to low signal strength at high $b$ values.

DSI data were reconstructed using a GQI approach (generalized $q$-sampling imaging) (Yeh et al., 2010); ODFs were reconstructed to 362 discrete sampling directions with a mean diffusion distance scaling factor of 1.2. The functional ROIs were projected back into the volume by performing the inverse of the volume-to-surface mapping procedure described above [and in the study by Greenberg et al. (2010)]. To make maximal contact with white-matter fibers, we grew ROIs toward the center of mass of the brain at each node by a distance equal to $50 \%$ of the cortex thickness between the gray/white and pial surfaces at that node. Once ROIs were in voxel units, we transformed them to the DSI space by applying the 12 point affine matrix generated when coregistering the surface-aligned anatomical image to the $\mathrm{b} 0$ image from the DSI data.

All fiber tracking was performed using DSIStudio (dsi-studio.labsolver. org). Currently no probabilistic tractography pipelines exist for DSI data, so a deterministic tractography approach was used. Rather than adopt a whole-brain tracking procedure, we used an ODF-streamlined ROIbased approach (Yeh et al., 2010). This maximizes the number of projections detected while ignoring irrelevant fiber pathways. Using random seeding, we initiated tracking, from each random position within the seed mask, in the direction of the most prominent fiber. Fiber progression continued with a step size of $0.5 \mathrm{~mm}$, minimum fiber length of 20 $\mathrm{mm}$, and turning angle threshold of $75^{\circ}$. To smooth each tract, the next directional estimate of each voxel was weighted by $80 \%$ of the previous moving direction and $20 \%$ by the forward direction of the fiber. Piloting has shown that $80 / 20 \%$ smoothing is a reasonable trade-off between anatomically plausible fiber tracts and good coverage of fibers. When smoothing $100 \%$ of the previous direction, results are no longer anatomically plausible because every fiber is essentially a straight line. However, smoothing of $60 \%$ or less reduces the number of fibers drastically due to the high contribution of noise. The tracking was terminated when the relative fractional anisotropy (FA) for the incoming direction exceeded a turning angle of $75^{\circ}$ or dropped below a preset FA threshold. Because signal-to-noise ratio is not consistent across individuals, we adopted a different FA threshold (range, 0.03-0.06) for each subject, to maximize fiber projections. For each individual, we began by tracking a set of control fibers from an anatomical ROI at the occipital pole with 500,000 seeds. We then adjusted the FA threshold for each subject so that the number of resulting fibers was equated ( \pm 100 fibers). Once tracked, all streamlines were saved in the TrackVis (www.trackvis.org) file format for visualization and/or further analysis in MATLAB.

In our connectivity analysis, we tracked fibers between pairs of ROIs in two passes (see Results). In the first pass, one ROI was seeded randomly 1 million times and fibers were tracked throughout the brain. Only fibers that connected to the second ROI were kept. In the second pass, the other ROI was seeded 1 million times and fibers were tracked to the previously seeded ROI. The resulting proportion of fibers (from 2 million random seeds) produced a measure of connectedness between these two regions. We used an iterative, constrained seeding (constant number of seeds in each tracking iteration) version of deterministic tractography to estimate degree of connectedness. Similar measures of "degree" have previously been used to provide stable results throughout the brain (Hagmann et al., 2008). To control for potential effects of distance between ROIs on connectedness values (distance bias), we scaled connectedness results by the corresponding distance between tracked ROIs. In each hemisphere, we measured the geodesic distances between pairs of IPS and visual cortex ROIs by first determining the central node of each surface-based ROI from the attention-mapping (IPS regions) and location-mapping (occipital regions) tasks. We then calculated distances along the mesh between node pairs and multiplied each individual connectedness value by these distances. This was done independently for each hemisphere to avoid smoothing of individual subject idiosyncrasies. The effect of this scaling is that fiber counts between longer distances are inflated (compensated for longer distance), while fiber counts between shorter distances are relatively reduced (penalized for shorter distance). We note that this metric is one of many ways to account for a distance bias in the data, and our choice may not be the best possible solution to this problem. Nevertheless, we believe geodesic distance scaling is a reasonable approach. However, it is far outside the scope of this paper to undertake an optimization of distance scaling, and we hope that others might perform this task in the future. To assess significance, scaled connectedness values were submitted to a three-way ANOVA with two levels of IPS region (aIPS, pIPS), three levels of visual area (V1, V2, V3/VP), and two levels of $z$-plane position (dorsal, ventral). Additional planned comparisons were tested.

In our fiber endpoint analysis, we tracked fibers between pairs of ROIs, in one direction. IPS map ROIs were seeded 2 million times and only fibers that connect to visual cortex ROIs (V1d, V2v, etc.) were retained. In TrackVis, these tracts were split into three sets of fibers, one for each of the three attention-mapped ROIs in pIPS (discarding fibers that did not contain an endpoint within these ROIs). Fiber endpoint locations in visual cortex were then transformed in MATLAB to the coordinate system of the surface ROIs to measure Euclidean distances to ROI centroids. By tracking fibers between larger map/visual cortex ROIs and comparing those endpoints to the centroids of the smaller location-specific ROIs, we have adopted a measure that is not biased by the choice of threshold $\alpha$ value (the centroid is a stable measure across a large range of $\alpha$ values). This endpoint-to-centroid distance measure captures the variability between fiber endpoints within the same bundle. An example is shown in Figure 5, where fiber endpoints from each of the three tracts in pIPS are shown plotted in surface coordinates, along with the borders of the corresponding surface ROIs. To assess significance, each set of endpoint distance values in each tract were submitted to separate two-way ANOVAs with three (or four) levels of tract selection ROI (lower, middle, upper; or lower, mid_d, mid_v, upper) and three (or four) levels of ROI centroid (lower, middle, upper; or lower, mid_d, mid_v, upper). Additional planned comparisons were also tested.

Finally, the dispersion index (DI) was calculated according to the following formula: $\mu_{\mathrm{v}} /$ mean $\left(\mu_{\mathrm{l}}, \mu_{\mathrm{m}}, \mu_{\mathrm{u}}\right)$, where $\mu_{\mathrm{v}}$ is the mean fiber end- 
point distance of one set of fiber endpoints in visual cortex, $\mu_{\mathrm{l}}, \mu_{\mathrm{m}}$, and $\mu_{\mathrm{u}}$ are the mean fiber endpoint distances in pIPS of the lower, middle, and upper contralateral locations, respectively. To assess significance, DI values were submitted to an omnibus two-way ANOVA with three levels of ROI subregion (lower, middle, upper) and three levels of visual cortex ROI (V1, V2, V3/VP). Middle dorsal and middle ventral subregion values were averaged in $\mathrm{V} 2$ and V3/VP to balance the design. Additional planned comparisons were also tested.

\section{Results}

\section{Behavioral and neural effects} during fMRI

Subjects completed three types of functional scans. During retinotopic mapping, subjects fixated a central dot that randomly changed color between white and black. Subjects were required to hold down response buttons when the dot turned white and release them when it was black (task-tocolor mapping counterbalanced across individuals). This task served to engage a narrow attentional focus on a single foveal location, ensuring that resulting bottom-up (stimulus-driven) activations in visual cortex were not contaminated by attention to the stimulus. Independent of fixation color changes, a "bowtie"-shaped stimulus of contrast reversing checkerboard alternated between stimulation of the horizontal and vertical meridians. Figure 1 shows a diagram of the displays as well as results of the contrast between epochs of horizontal and vertical meridian stimulation on a left hemisphere spherical surface representation of a typical brain from our group. This method allowed us to map the borders between retinotopic regions of visual cortex (Slotnick and Yantis, 2003), as shown by the thin colored lines in Figure 1. We then drew rectangular regions of interest, filling the space between these borders, for V1d, V1v, V2d, $\mathrm{V} 2 \mathrm{v}, \mathrm{V} 3 \mathrm{~d}$, and $\mathrm{VPv}$. Importantly, this task produced only minimal significant activation outside of the regions shown in Figure 1 (i.e., only retinotopic visual regions were activated).

This same task was used during the stimulus-based location-mapping experiment. In these scans, subjects fixated centrally while one of six RSVP streams of letters (Potter and Levy, 1969; Reeves and Sperling, 1986) appeared in the periphery. Only one RSVP stream was present at a time, rotating sequentially through all six streams, three times per run. Figure $2 a$ shows a diagram of the display and an example of discrete retinotopic ROIs in V1-V3 resulting from this experiment. Note the precise agreement between func-

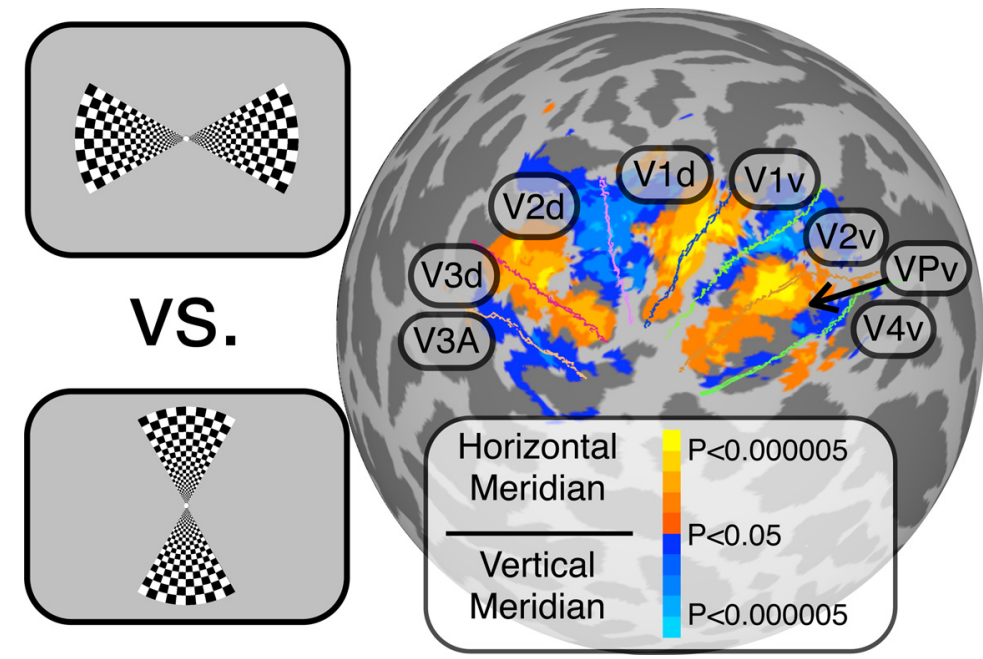

Figure 1. Retinotopic meridian-mapping results. Results of the retinotopic meridian-mapping experiment in the left hemisphere of a representative individual. The left side of the figure shows diagrams of the horizontal (top) and vertical meridian (bottom) stimuli. The activation shown on the spherical surface was generated by contrasting epochs of these two conditions. The warm colors indicate significant nodes for horizontal, and the cool colors indicate significant nodes for vertical; maps are thresholded at $p<0.05$, corrected. The colored lines in the surface are hand-drawn borders between visual areas, which follow the ridge of maximal activation. Visual areas are also labeled.

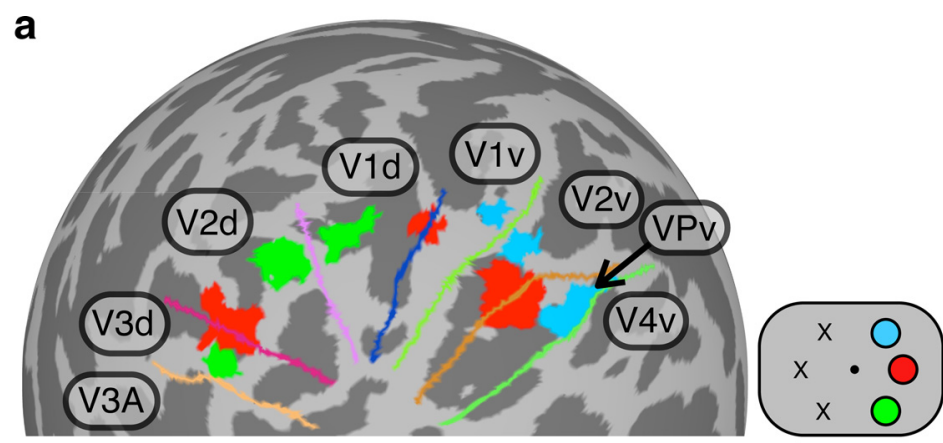

b

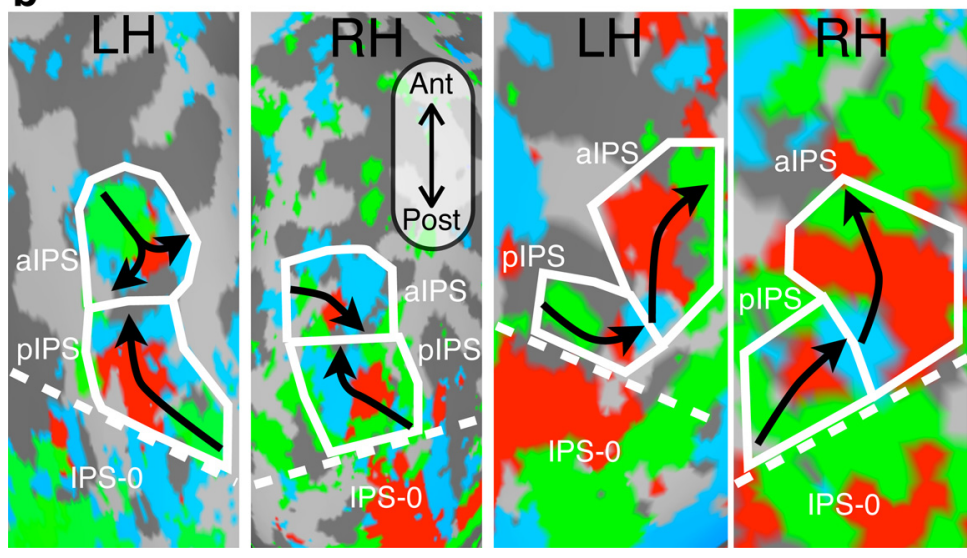

Figure 2. Location-mapping and attention-mapping results. A diagram depiction (top right) showing placeholders for each of the six RSVP stream locations in the location-mapping and attention-mapping tasks. Colors in the diagram are consistent in the functional maps. $\boldsymbol{a}$, Left hemisphere spherical surface from a representative individual showing the borders between visual cortex regions (from the meridian-mapping experiment) and the activation from the stimulus-based location-mapping experiment in V1-V3/VP. Theseactivations are from right visual field stimuli, which became functional ROIs for subsequent analyses. $\boldsymbol{b}$, Two left hemisphere and two right hemisphere surface representations of the posterior portion of the IPS showing functional activation from the attention-mapping experiment colored in accordance with the diagram in $\boldsymbol{a}$. The dotted lines indicate the anterior borders of significant activation from the meridian-mapping experiment. The solid white lines indicate an estimate of visuotopic attention map boundaries for posterior and anterior IPS. The black arrows show the visuotopic orientation progression from lower visual field to upper visual field representations. Known visuotopic orientation patterns suggest that the posterior map (pIPS) corresponds to IPS-1 and the anterior map (aIPS) corresponds to IPS-2. The map present posterior to the dotted line, in retinotopic cortex, is labeled by its likely region (IPS-0). 
Table 1. Brain regions mapped in IPS from the attention-mapping task for a representative individual

\begin{tabular}{llllll}
\hline & & & \multicolumn{3}{c}{$\begin{array}{l}\text { Talairach coordinates } \\
\text { (center of mass) }\end{array}$} \\
\cline { 5 - 7 } Corresponding RSVP location & IPS map & Hemisphere & $x$ & $y$ & $z$ \\
\hline Lower right & IPS-2 & L & -45 & -66 & 46 \\
Middle right & IPS-2 & L & -32 & -67 & 54 \\
Upper right & IPS-2 & L & -26 & -67 & 54 \\
Lower right & IPS-1 & L & -34 & -77 & 33 \\
Middle right & IPS-1 & L & -31 & -75 & 33 \\
Upper right & IPS-1 & L & -27 & -81 & 42 \\
Lower left & IPS-2 & R & 37 & -75 & 43 \\
Middle left & IPS-2 & R & 29 & -74 & 43 \\
Upper left & IPS-2 & R & 21 & -81 & 46 \\
Lower left & IPS-1 & R & 24 & -88 & 40 \\
Middle left & IPS-1 & R & 25 & -84 & 30 \\
Upper left & IPS-1 & R & 28 & -79 & 29 \\
\hline & & & & &
\end{tabular}
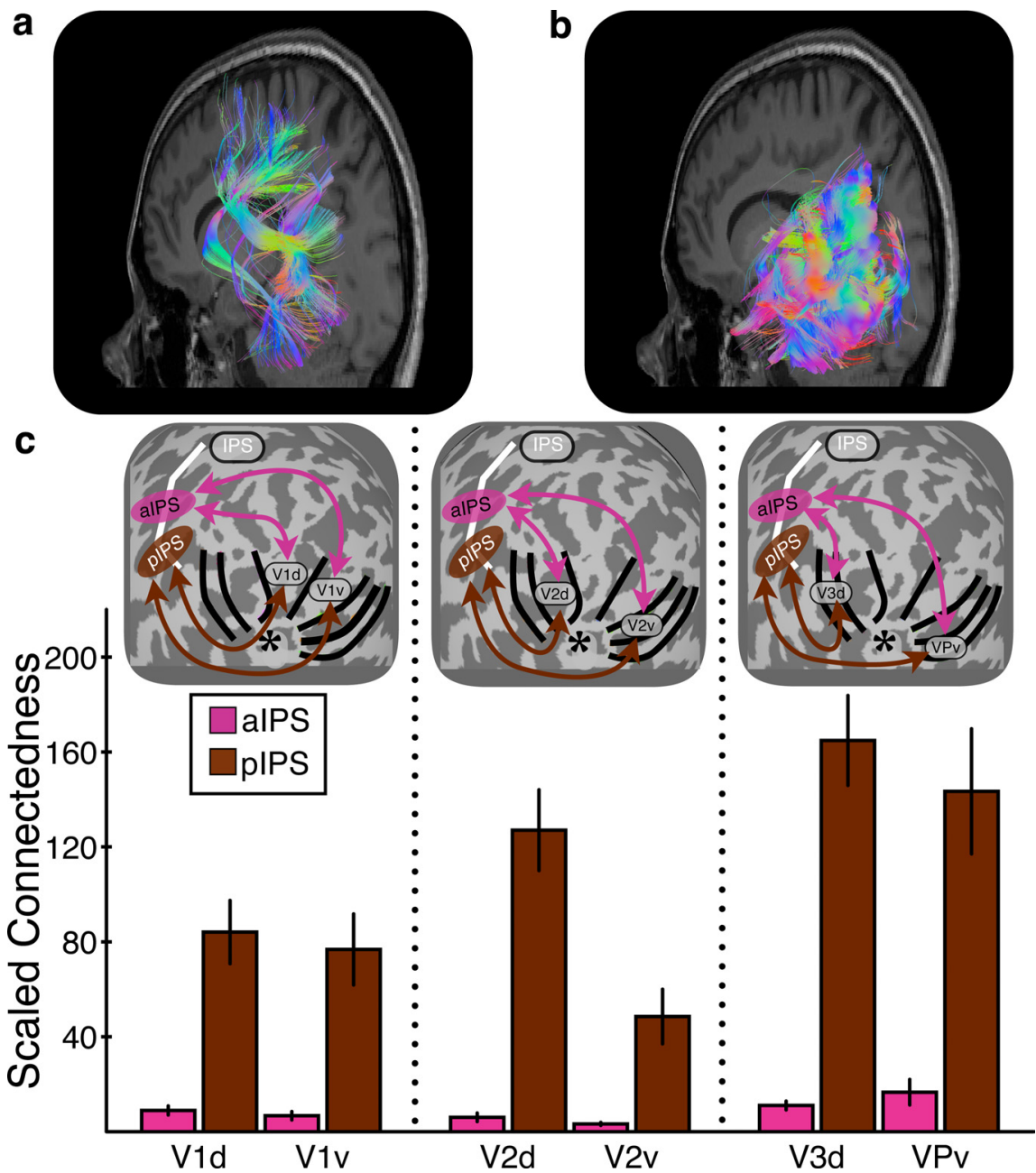

Figure 3. Connectedness between IPS and visual cortex. $\boldsymbol{a}$, Fibers connecting aIPS and all six regions in visual cortex ( $10 \%$ of total fibers shown) in the left hemisphere of one subject. $\boldsymbol{b}$, Fibers connecting pIPS and all six regions in visual cortex ( $10 \%$ of total fibers shown) in the same hemisphere displayed in a.c, The scaled (by ROI distance) percentage of 2 million seeds per ROI pair that produced fibers is plotted for both pIPS and aIPS. While pIPS shows connectivity with each visual cortex ROI, aIPS has consistently very little connectivity. An overall linear increase in connectedness from V1 through V3 can be seen. Diagram depictions of cortex show ROls tested, centered above corresponding data in the graph. The colored arrows correspond to bar colors in the graph. The asterisks indicate approximate location of the occipital pole. Error bars in graph are SEM across the 10 hemispheres tested. of our middle (red) ROI that straddle the border between these two regions (V2 and V3/VP).

In the attention-mapping experiment, subjects fixated centrally while covertly attending to one of the six RSVP streams occupying the same spatial positions as in the location-mapping experiment. During these runs, all six streams were present stream for a red target digit (among otherwise white letters). Subjects were instructed to categorize the digit as odd or even, indicated by button press, and then shift attention to the next out trouble (mean proportion no-response, $0.006 \pm 0.005$ ) and shifted attention as instructed on virtually all trials. Although this was a challenging task, subjects performed significantly above chance on digit categorization (mean accuracy, $0.82 \pm 0.05 ; t_{(4)}=4.43 ; p=0.01$ ).

Figure $2 b$ shows four example hemispheres of the resulting functional activation in IPS. Although neither firm anatomical landmarks nor robust criteria exist for identifying posterior parietal cortex (PPC) attention topographic maps, we were able to detect two such maps in each hemisphere by identifying continuous contralateral attention patterns in the posterior IPS that did not overlap with the activations generated by either of the other two experiments outlined above. This heuristic ensured that the IPS ROIs were restricted to regions outside retinotopic visual cortex, thus staying anterior/dorsal to the IPS-0 map (for nomenclature, see Silver and Kastner, 2009). Previous reports of topography in this region show that IPS-1 generally represents (posteriorly to anteriorly) the lower to upper vertical meridians, whereas IPS-2 represents the upper to lower vertical meridians, with a reversal occurring at the border between these two maps. This is consistent with the visuotopic orientations shown in Figure $2 b$ (black arrows). Note that, in the two left panels, there is a gap between the two regions of activation associated with our upper field stimulus (blue), at the border between pIPS and aIPS. This silent region is likely dedicated to a representation of the upper vertical meridian, which we did not stimulate in this experiment. The data in Figure $2 b$ suggest that our pIPS maps correspond to IPS-1 and our aIPS maps correspond to IPS-2. Table 1 contains Talairach coordinates (Talairach and Tournoux, 1988) of IPS ROIs for a representative individual; locations are also in agreement with the published literature (Silver et al., 2005; Swisher et al., 2007). tional ROIs and visual area borders, despite being generated from independent data. Importantly, V2 and V3/VP contain a representation of the horizontal meridian in both dorsal and ventral subregions, which can be seen in Figure $2 a$ as instances

\section{Structural connectivity between IPS and visual cortex}

In each hemisphere, we defined a pIPS and an aIPS "map ROI" that circumscribed the respective attention-defined ROIs in posterior and anterior IPS, respectively, which were generated from 
the attention-mapping experiment (Fig. 2b). Each map ROI included only the three attention-defined ROIs and the nonactivated nodes between them, to create a contiguous region. By identifying spatially identical regions in retinotopic and topographically organized PPC regions, we were able to ensure that fibers tracked precisely between directly corresponding representations of visual space. For each visual cortex ROI created from the meridian-mapping experiment, we measured the structural connectivity to both pIPS and aIPS. We used deterministic tractography to randomly seed an IPS map ROI 1 million times and generate fibers connecting to the region in visual cortex being tested. We then applied the reverse mapping, seeding the visual cortex ROI 1 million times and tracking fibers connecting to the IPS region being tested (see Materials and Methods). This metric (also referred to as "degree") has been used to depict the connectedness between regions of cortex (Hagmann et al., 2008). With deterministic tractography, this reflects the ease with which connections between two regions can be mapped (see Materials and Methods). Figure 3 shows fiber streamlines from a single hemisphere between all six visual cortex regions and both aIPS (Fig. $3 a$ ) and pIPS (Fig. $3 b$ ) ROIs; $10 \%$ of all streamlines are shown in each image. Although aIPS tracts cover a larger region of cortex (due to map ROI locations) one can see the disparity in connectedness, with seeds producing many fewer fibers from aIPS (3958 shown) than from pIPS (124,769 shown). The very close proximity between aIPS and pIPS prevented us from performing direct tractography between these two regions. Fiber-tracking methods, although becoming increasingly refined, have great difficulty tracking streamlines between nearby locations in cortex. This is likely due to the sharp curvature of the U-shaped fibers that would connect such close areas or that such adjacent connections may go through layer 1 projections, which are not visible with modern diffusion imaging approaches. A potential confound of tractography methods is a distance bias, such that two nearby regions are more likely to produce more tracts than two distant regions. As a method of controlling for potential distance effects, all reported connectedness values have been scaled by the distance between tracked ROIs (for details, see Materials and Methods). Figure $3 c$ shows the percentage of 2 million seeds per ROI pair that produced fibers (scaled by the distance between tracked ROIs), for each IPS-visual cortex pairing. This analysis yielded the following important results.

First, while pIPS was highly connected with each of the six visual cortex regions tested, aIPS had significantly fewer connections with visual cortex overall $\left(F_{(1,9)}=\right.$ 19.25; $p=0.002)$. Since we scaled our connectedness values by ROI distance, this effect is unlikely to simply be a function of distance. There may, in fact, be a residual distance bias in the data, but the result of our method of correction (scaling by geodesic distances) suggests that distance is not contributing in a major way. Second, we found no overall effect of IPS connectivity as a function of either visual cortex area $\left(\mathrm{V} 1, \mathrm{~V} 2, \mathrm{~V} 3 / \mathrm{VP} ; F_{(2,18)}=3.35 ; p=0.07\right)$ or subregion of visual cortex (dorsal vs ventral; $F_{(1,9)}=1.42 ; p=0.263$ ). Third, while the interaction of connectivity between IPS region and visual cortex region was marginally significant $\left(F_{(2,18)}=3.37 ; p=0.057\right)$,
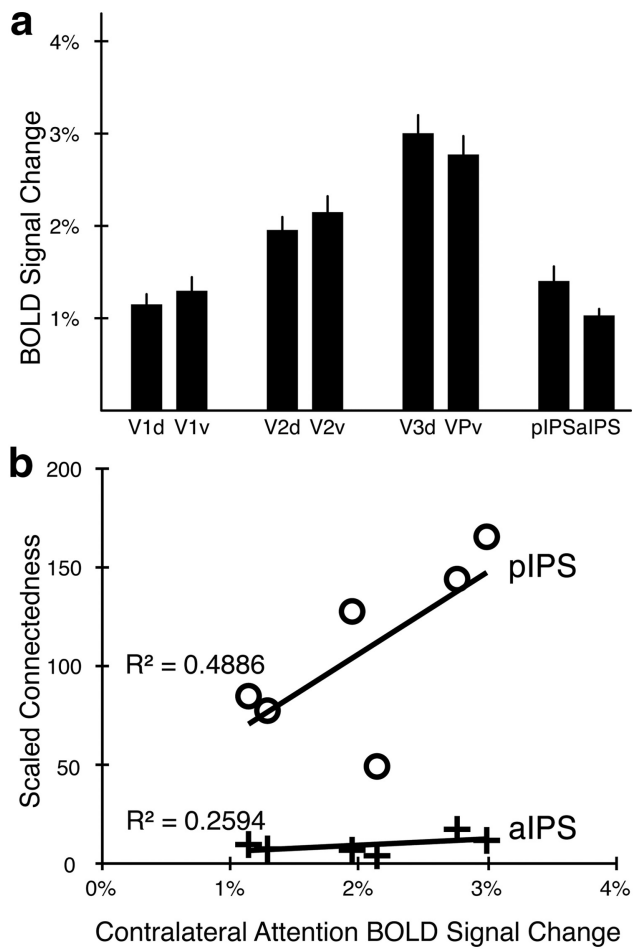

Figure 4. Attentional modulation and correlation with connectedness. $\boldsymbol{a}, \mathrm{BOLD}$ signal during epochs of attention to the contralateral visual field stimuli during the attention-mapping experiment in map ROIs ( 2 in IPS) and visual cortex ROIs (6 in occipital lobe). Signal increases linearly from V1 through V3. IPS regions show similar activation levels to V1. Error bars are SEM across the 10 hemispheres tested. $\boldsymbol{b}$, Correlation between attention BOLD signal and scaled connectedness for pIPS and aIPS, separately. pIPS shows a strong positive linear relationship, while alPS shows only a weak relationship. back to surface coordinates to show the correspondence between endpoints and functional ROIs in pIPS. Individual endpoints are shown as small spheres, colored according to the ROl in which the seed was placed to generate that fiber. Endpoint colors match colors of functional ROls from the attention-mapping experiments painted on the underlying gray/white boundary surface in a representative left hemisphere. Note that the ROIs used for tractography seeding extended out on both sides of the surface (see Materials and Methods), which is why many of the endpoints appear to lie above the surface, instead of on it. Legend at left indicates the location of detail in posterior parietal cortex (Lat, lateral; Med, medial; Ant, anterior; Post, posterior).

planned comparisons revealed that connectedness with pIPS varied as a function of visual area $\left(F_{(2,18)}=3.42 ; p=0.05\right)$, while this was not true for aIPS $\left(F_{(2,18)}=1.39 ; p=0.275\right)$. Specifically, pIPS scaled connectedness to V1 was relatively modest, stronger for $\mathrm{V} 2$, and strongest for V3. We explored the functional correlate of this result by examining the BOLD signal change in these regions during the attention-mapping task. 


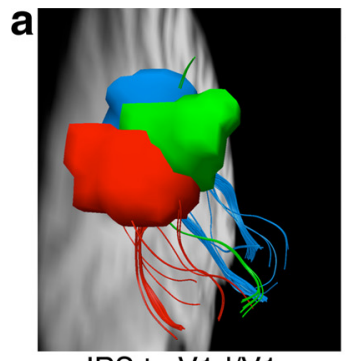

IPS to V1d/V1v

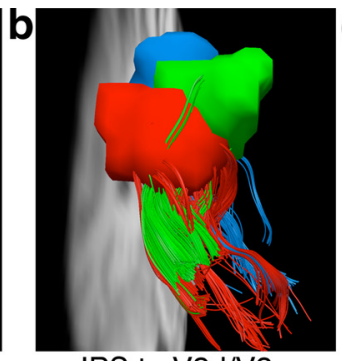

IPS to $\mathrm{V} 2 \mathrm{~d} / \mathrm{V} 2 \mathrm{~V}$

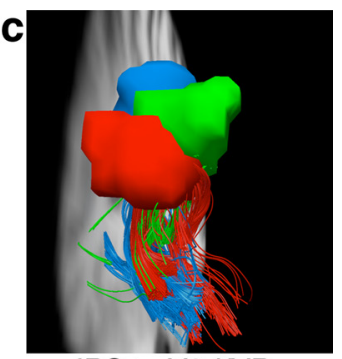

IPS to V3d/VPV

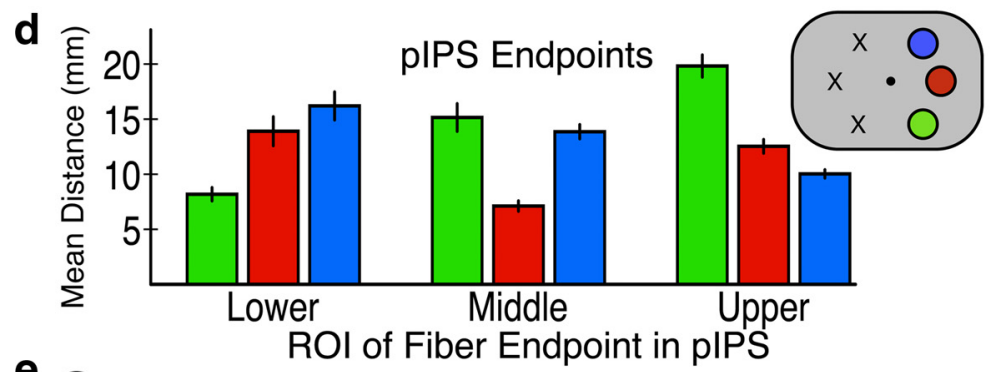

है
है
0
$\frac{0}{\sigma}$
$\frac{\pi}{0}$
$\frac{.0}{0}$
$\frac{1}{\pi}$
$\stackrel{0}{\Sigma}$

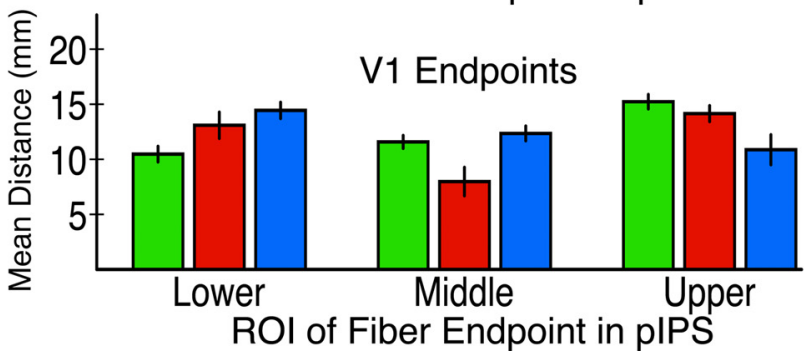

Figure 6. Endpoint distance to ROI centroids. Fibers tracked from attention ROls in pIPS to V1 (a), V2 (b), and V3/VP (c) in a representative hemisphere are colored according to the ROI from which they were seeded. Note the relative increase in IPS tracts in $\boldsymbol{a}$ versus $\boldsymbol{b}$, and $\boldsymbol{b}$ versus c. In addition, the interdigitation (or dispersion) of the tracts increases along the same continuum. $\boldsymbol{d}$, The mean Euclidean fiber endpoint distance to each attention mapped ROI centroid in pIPS, for fibers tracked to V1. The $x$-axis indicates the pIPS subregion of each tract; colors correspond to ROI centroids from which fiber endpoint distance measurements were computed. Each set of fiber tracts is nearest to its corresponding ROI centroid (i.e., lower fibers are closest to lower ROI centroid).e, The mean fiber endpoint distance to each location mapped ROI centroid in V1. The pattern of endpoint distances is largely unchanged from $\boldsymbol{d}$. Error bars in both graphs are SEM across 10 hemispheres tested.

Table 2. Statistical analysis of fiber endpoint distance measurements in pIPS

\begin{tabular}{lrrrl}
\hline & Lower & Middle & Upper & Ftest \\
\hline $\begin{array}{l}\text { pIPS-V1 tract } \\
\text { pIPS ROI centroid }\end{array}$ & & & & \\
$\quad$ Lower & $\mathbf{8 . 1 8}$ & 15.15 & 19.82 & $F_{(2,18)}=38.88 ; p<0.0001$ \\
$\quad$ Middle & 13.90 & $\mathbf{7 . 1 1}$ & 12.53 & $F_{(2,18)}=10.40 ; p=0.001$ \\
$\quad$ Upper & 16.20 & 13.86 & $\mathbf{1 0 . 0 3}$ & $F_{(2,18)}=3.67 ; p=0.046$ \\
pIPS-V2 tract & & & & \\
$\quad$ PIPS ROI centroid & & & & \\
$\quad$ Lower & $\mathbf{9 . 0 4}$ & 15.92 & 20.17 & $F_{(2,18)}=26.36 ; p<0.0001$ \\
$\quad$ Middle & 14.80 & $\mathbf{7 . 1 4}$ & 12.09 & $F_{(2,18)}=8.45 ; p=0.003$ \\
$\quad$ Upper & 16.96 & 12.22 & $\mathbf{7 . 0 7}$ & $F_{(2,18)}=6.42 ; p=0.013$ \\
pIPS-V3/VP tract & & & & \\
pIPS ROI centroid & & & & \\
$\quad$ Lower & $\mathbf{9 . 9 4}$ & 15.23 & 18.32 & $F_{(2,18)}=14.77 ; p<0.0001$ \\
$\quad$ Middle & 14.91 & $\mathbf{8 . 8 6}$ & 11.43 & $F_{(2,18)}=8.85 ; p=0.002$ \\
$\quad$ Upper & 17.84 & 12.86 & $\mathbf{8 . 4 2}$ & $F_{(2,18)}=23.29 ; p<0.0001$ \\
\hline
\end{tabular}

Values are expressed in millimeters. The bold values highlight the minimum distance for a given centroid when the corresponding $F$ value is significant.

\section{Correlation with BOLD signal change}

For each of the eight ROIs described above (six regions of visual cortex and two map ROIs in IPS), we measured the average BOLD percentage signal change during epochs of attention to the contralateral visual field in the attention-mapping experiment.
We observed significant activation in each ROI (Fig. $4 a$; all values of $t_{(9)}>4$; all values of $p<0.003)$. For the visual cortex regions (V1d, V2v, etc.), we found no differences in attentional modulation between dorsal and ventral subregions of visual cortex $\left(F_{(1,9)}=0.032 ; p=0.863\right)$. In IPS, the signal change in pIPS trended greater than in aIPS $\left(t_{(9)}=2.07 ; p=0.06\right)$. Furthermore, attentional modulation increased significantly from V1 to V2 to V3 (linear trend, $F_{(1,9)}=33.06 ; p=0.0003$ ), which mirrors the structural connectivity pattern reported above. To examine this further, we computed the correlation between the attentional modulations in visual cortex and scaled connectedness with IPS. We found a striking difference between the two IPS regions tested (Fig. $4 b$ ). Linear least-squares trend lines through each dataset account for different proportions of variance $\left(R^{2}\right.$ of 0.49 and 0.26 for pIPS and aIPS, respectively), and the PIPS slope is significantly different from zero $\left(m=41.45 ; t_{(4)}=114.82 ; p<0.0001\right)$, whereas the aIPS slope is not $(m=0.316$; $\left.t_{(4)}=0.60 ; p=0.578\right)$. Thus, the relationship between connectedness and attentional modulation in visual cortex is strongly positive for pIPS and absent for aIPS. These results suggest that posterior IPS may be the gateway through which attentional modulations of visual cortex pass.

\section{Consistency of fiber endpoint visuotopy}

We next examined the consistency of topographic segregation in fiber streamlines that connect IPS and visual cortex. The three attention-mapped ROIs identified in pIPS were used to generate individual white-matter tract estimates for each of the six ROIs in visual cortex, yielding 18 sets of tracts per hemisphere. We then mapped the two endpoints of each fiber back into the space of the fMRI data. Figure 5 shows an example of a representative hemisphere in which functional ROIs in pIPS are rendered on the surface in colors corresponding to the stimulus diagram. The fiber endpoints are indicated by small spheres, colored according to the pIPS ROI from which each fiber originated. These tracts were generated because they contained one endpoint in a location-specific ROI in pIPS and the other endpoint in a meridian-mapped visual cortex ROI. That is, no constraint was introduced as to the visuotopy of the endpoints in visual cortex, making this an unbiased measure of topographic consistency in visual cortex. Figure $6 a-c$ shows examples of these tracts for one participant in $\mathrm{V} 1, \mathrm{~V} 2$, and V3/VP, respectively. Note the relative increase in tracts and fiber interdigitation (or dispersion) as one moves from V1 to V2 to V3/VP.

We measured the distance of each fiber endpoint from the centroid of the three discrete, location-mapped ROIs in each region of visual cortex and in pIPS. Figure $6 d$ and Table 2 show the mean distance of fiber endpoints to the centroid of each of the three pIPS attention-mapped ROIs, for pIPS-to-V1 tracts. In each case, the fibers from each of the three pIPS ROIs are closest 
Table 3. Statistical analysis of fiber endpoint distance measurements in visual cortex

\begin{tabular}{|c|c|c|c|c|c|c|}
\hline & Lower & Middle & Mid_d & Mid_v & Upper & Ftest \\
\hline \multicolumn{7}{|l|}{ pIPS-V1 tract } \\
\hline \multicolumn{7}{|c|}{ V1 R0I centroid } \\
\hline Lower & 10.47 & 11.58 & - & - & 15.23 & $F_{(2,18)}=5.61 ; p=0.013$ \\
\hline Middle & 13.09 & 7.98 & - & - & 14.15 & $F_{(2,18)}^{(2,1)}=4.16 ; p=0.033$ \\
\hline Upper & 14.44 & 12.35 & - & - & 10.87 & $F_{(2,18)}=3.70 ; p=0.045$ \\
\hline \multicolumn{7}{|l|}{ pIPS-V2 tract } \\
\hline \multicolumn{7}{|c|}{ V2 ROI centroid } \\
\hline Lower & 12.37 & - & 12.82 & 21.41 & 22.48 & $F_{(3,27)}=13.31 ; p<0.0001$ \\
\hline Mid_d & 12.35 & - & 14.88 & 22.96 & 22.68 & $F_{(3,27)}=2.17 ; p=0.115$ \\
\hline Mid_v & 24.06 & - & 24.83 & 11.86 & 8.79 & $F_{(3,27)}^{(3,27)}=31.77 ; p<0.0001$ \\
\hline Upper & 24.83 & - & 25.70 & 9.22 & 8.59 & $F_{(3,27)}=23.30 ; p<0.0001$ \\
\hline \multicolumn{7}{|c|}{ pIPS-V3/VP tract } \\
\hline \multicolumn{7}{|c|}{ V3/VP ROI centroid } \\
\hline Lower & 16.18 & - & 15.73 & 21.42 & 21.89 & $F_{(3,27)}=3.68 ; p=0.024$ \\
\hline Mid_d & 15.57 & - & 16.53 & 21.46 & 22.37 & $F_{(3,27)}=0.254 ; p=0.857$ \\
\hline Mid_v & 25.69 & - & 24.97 & 12.95 & 9.65 & $F_{(3,27)}^{(3,27)}=49.13 ; p<0.0001$ \\
\hline Upper & 25.97 & - & 25.71 & 10.07 & 10.33 & $F_{(3,27)}=57.41 ; p<0.0001$ \\
\hline
\end{tabular}

Values are expressed in millimeters. The bold values highlight the minimum distance for a given centroid when the corresponding $F$ value is significant.

to the center of the ROI from which they were selected, and further from the other two ROIs. For example, the lower ROI fibers (shown on the left of the graph) have the shortest mean distance to the lower ROI centroid (shown in green) in pIPS. This distance is significantly longer to the middle centroid (red; $t_{(9)}=$ $3.29 ; p=0.009$ ) and longest to the upper centroid (blue; $t_{(9)}=$ $4.24 ; p=0.002)$. The same pattern was seen for fibers tracked to $\mathrm{V} 2$ and V3/VP (Table 2). These values provide a measure of the baseline "spread" of the topographic relationship among fibers within pIPS.

We then examined the mean fiber endpoint distances in visual cortex, at the other end of these tracked fibers. Mirroring the pIPS endpoint results, Figure $6 e$ and Table 3 show that pIPS-to-V1 fiber endpoints in V1 respect the visuotopic location from which they were selected. For example, the V1 fibers that had parietal endpoints in the lower pIPS ROI (on the left of the graph) are closest to the lower V1 ROI centroid (green), further from the middle centroid (red), and significantly farthest from the upper centroid (blue; $t_{(9)}=2.78 ; p=0.021$ ). This key result shows a topographic consistency in the fibers between pIPS and V1, providing compelling evidence that there exist direct connections between these topographically mapped regions. The data in $\mathrm{Ta}-$ bles 2 and 3 show that the topographic consistency within V1 tracts was degraded for $\mathrm{V} 2$ and $\mathrm{V} 3 / \mathrm{VP}$ tracts. In these downstream regions, while tracts were topographically segregated in pIPS, they became more dispersed (as measured by mean distance to topographically corresponding regions) when they terminated in V2 and V3/VP.

To summarize the endpoint distance findings, we devised a simple metric for characterizing the relative spread of topographic fibers in pIPS versus visual cortex. The DI is a ratio of the mean fiber endpoint distance from ROI centroids in visual cortex to the mean fiber endpoint distance from ROI centroids in pIPS. This value is calculated separately for each set of fiber endpoints in each region of visual cortex. A DI close to 1.0 indicates that the two endpoints for a set of fiber tracts were equally dispersed from topographic ROIs in both pIPS and visual cortex; whereas a DI different from 1.0 suggests a discrepancy between the topographic organization in pIPS and in visual cortex. Values $>1.0$ signify more topographic dispersion in visual cortex compared with the baseline spread of those fibers measured in pIPS. Values

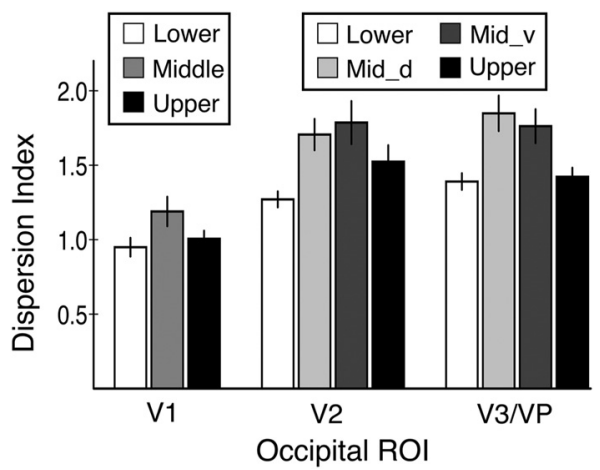

Figure 7. Dispersion index. DI values for each set of fiber endpoints in visual cortex (for exact calculation, see Materials and Methods). DI is a measure of the relative endpoint topographic dispersion in visual cortex versus IPS. A DI of 1.0 suggests little difference between fiber endpoint dispersion in IPS and visual cortex (such as in V1). A DI near 1.6 (such as in V2 and V3/VP) suggests that endpoints are $\sim 60 \%$ less topographic in visual cortex compared with IPS. Mid_d, Fibers that track to the dorsal visual cortex representation of the middle stimulus (along the horizontal meridian); Mid_v, fibers for ventral representation of middle stimulus. The left legend applies to V1 data only; the right legend applies to V2 and V3/VP data. Error bars represent SEM.

$<1.0$ indicate that endpoints are more topographically organized in visual cortex than they are in pIPS. Figure 7 contains a graph of DI values for all fiber tracts and shows that fiber endpoints in V1 have a DI near 1.0 (range, 0.95-1.19). However, in V2 and V3/VP, the DI is centered near 1.6 (V2 mean, 1.57; V3 mean, 1.61), suggesting that fibers that connect to $\mathrm{V} 2 / \mathrm{V} 3$ are less topographically organized, or 60\% more dispersed (V2 range, 1.27-1.79; V3/VP range, $1.39-1.85$ ) than those that track to primary visual cortex. The DI ANOVA yielded a significant main effect of visual area $\left(\mathrm{V} 1, \mathrm{~V} 2, \mathrm{~V} 3 ; F_{(2,18)}=6.85 ; p=0.006\right) ; \mathrm{V} 1$ had a lower DI than either V2 $\left(t_{(9)}=2.82 ; p=0.020\right)$ or V3 $\left(t_{(9)}=2.93 ; p=0.017\right)$, which did not differ $\left(t_{(9)}=0.239 ; p=0.816\right)$. There was also a significant main effect of subregion (lower, middle, upper; $F_{(2,18)}=$ $8.00 ; p=0.003)$, and the interaction was not significant.

\section{Discussion}

Using diffusion-weighted fiber tracking in parallel with functional brain imaging, we have shown that there exist direct topographic white-matter connections between IPS and visual cortex that may subserve the control of visuospatial selective attention in humans. Posterior IPS is more highly connected with visual cortex than a more anterior region of IPS, and pIPS connectedness showed a strong positive correlation with increasing effects of attention on BOLD signal from V1 to V3. Finally, the pattern of topographic connectivity is well preserved between pIPS and visual cortex. Nevertheless, downstream visual regions maintain the spatially specific mapping with IPS less precisely than V1, where there is essentially no difference in fiber endpoint dispersion compared with IPS.

The biased competition model of visual attention suggests that direct axonal connections exist between attentional control regions and visual cortex; these control regions serve as the source of a biasing signal that resolves competition between multiple stimuli represented within the receptive fields of retinotopic visual cortex (Desimone and Duncan, 1995). The connections identified in the current study provide a candidate set of white-matter fibers that are ideally suited to mediate biased competition by way of the topographic organization at both ends of the tracts. The correlation between contralateral attention BOLD activation and connectedness suggests that these fibers carry visuotopic attention signals. 
Before the current work, evidence in support of the topographic connectivity hypothesis between PPC and visual cortex was only indirect. For example, activity in these two regions is functionally correlated, suggesting that attention enhances the signal coherence between IPS and visual cortex (Lauritzen et al., 2009; Uddin et al., 2010). Additionally, known direct structural projections connect posterior IPS to visual cortex in macaque (Baizer et al., 1991; Lewis and Van Essen, 2000). Since monkey PPC contains a comparable map of spatial attention to that of humans (Bisley and Goldberg, 2003), and given that monkey and human IPS have similar functional-anatomical homologies (Grefkes and Fink, 2005; Arcaro et al., 2011; Mars et al., 2011), one might have thought it likely that human IPS may similarly be highly interconnected with visual cortex. The present study is the first investigation of human structural connectivity leveraging the mirrored topographic organization of IPS and visual cortex, to test this hypothesis directly.

We explored the structural connectivity between corresponding topographic regions of IPS and visual cortex in humans. Recent diffusion-weighted MR imaging has revealed a parcellation between anterior and posterior IPS connectivity, with anterior regions linked to the motor and eye movement systems in prefrontal cortex, and posterior IPS connected to the visual system in occipital cortex (Uddin et al., 2010). The current investigation examined whether the connectivity pattern between these regions respects the corresponding visuotopic organization and the degree to which these white-matter connections underlie attentional control of early visual representations.

We found that attention modulations in visual cortex were correlated with connectedness to pIPS; however, attention modulations in aIPS were only weakly correlated with connectedness. Nevertheless, aIPS was identified as topographic in our attentionmapping task and was only slightly less modulated by attention to contralateral visual field locations than pIPS. Therefore, one may ask what role aIPS plays in attentional control. There are at least three possibilities. First, aIPS may serve as a conduit through which pIPS connects to the rest of the frontoparietal attention network. Recent neuroimaging studies have provided evidence for several subprocesses of attentional control in distinct parts of the frontoparietal network such as orienting (superior parietal lobule) (Greenberg et al., 2010), distracter filtering (middle/inferior frontal gyri) (Leber, 2010) (A. Greenberg, S. Friedman-Hill, L. Pessoa, L. Ungerleider, unpublished observations), and coupling between attention and eye movements (frontal eye fields) (Kelley et al., 2008; Van Ettinger-Veenstra et al., 2009). If pIPS is to carry spatial biasing signals to visual cortex, it must have access to the results of computations concerning these other aspects of attentional control; aIPS may be the region through which that information propagates to pIPS. Second, aIPS might be the human homolog of macaque area VIP, a multimodal attention region of IPS, anterior and medial to LIPv. In monkeys, this region shows some topography and is connected to large portions of the somatosensory, vestibular, oculomotor, auditory, and visual systems, including LIPv (Lewis and Van Essen, 2000), and is possibly involved in eye-hand coordination. Third, aIPS may exhibit a stronger organization along nonspatial dimensions such as color or motion. This would imply a high degree of connectivity with parts of the visual system specialized for these features such as $\mathrm{MT}+$ (motion) and V4 (color), therefore playing a similar role in the attention network to pIPS, but in a nonspatial domain. Recent fMRI evidence has shown heterogeneity in PPC for the control of attention in different domains (Greenberg et al., 2010), which is consistent with this latter proposal.
At first glance, the connectivity results reported here may seem incompatible with the topographic dispersion results. On the one hand, connectedness between pIPS and visual cortex increases from V1 through V3. By contrast, the systematicity of visuotopy in these fiber tracts decreases from V1 through V3. However, one must consider the different roles that V1, V2, and V3/VP play in the visual hierarchy. Specifically, at the eccentricity we stimulated $\left(5^{\circ}\right)$, population receptive field $(\mathrm{pRF})$ sizes have been shown to approximately triple from V1 $\left(\sim 0.5^{\circ}\right)$ to $\mathrm{V} 3 / \mathrm{VP}$ $\left(\sim 1.5^{\circ}\right)$ (Dumoulin and Wandell, 2008). Therefore, items in the visual field to which attention will be deployed will only rarely be small enough to fall entirely within a V1 pRF, and thus are unlikely to engage in competition with other stimuli for representation at that level of the visual system (Desimone and Duncan, 1995). Competition is much more likely to take place further downstream where pRFs are larger, thus accounting for the increasing effects of attention from V1 through $\mathrm{V} 3$, as we show here and has been reported previously (Silver et al., 2005; Serences and Yantis, 2007). Thus, the connectivity must support the need for more attention throughput to V3 than to V1. However, the precision of small RFs in V1 for selecting a restricted spatial location lends itself to more systematic visuotopic connections with pIPS. PPC contains a detailed map of attentional priority (Bisley and Goldberg, 2003, 2006) and therefore must maintain spatial precision when communicating with a precise retinotopic region, such as V1. Yet, for downstream regions with larger RFs (leading to less precise retinotopy), the connectivity is naturally less systematic and more topographically dispersed and redundant.

In summary, the performance advantage observed when attention is deployed to a region of space before stimulus onset relies on a number of still poorly understood neural mechanisms. However, a key component is the communication between attentional control regions (in IPS) and sensory cortex where visual space is represented. We have identified white-matter structures that are ideally suited to carry biasing signals in visuotopic coordinates from control regions to sensory regions. These regions vary in connectedness with the amplitude of attentional modulations, and vary in topographic consistency with the need for spatial precision in each visual area. The current data provide critical evidence for the biased competition theory of attention in humans and specify neurobiological constraints on the functional brain organization of visual attention. These results lay the foundation for future efforts to decode how selective processing of visual information is controlled by top-down attentional modulation. Furthermore, given the multimodal nature of PPC more generally, these findings suggest that other sensory modalities may be similarly connected to control regions via an inherent, modality-specific organization.

\section{References}

Arcaro MJ, Pinsk MA, Li X, Kastner S (2011) Visuotopic organization of macaque posterior parietal cortex: a functional magnetic resonance imaging study. J Neurosci 31:2064-2078.

Baizer JS, Ungerleider LG, Desimone R (1991) Organization of visual inputs to the inferior temporal and posterior parietal cortex in macaques. J Neurosci 11:168-190.

Bashinski HS, Bacharach VR (1980) Enhancement of perceptual sensitivity as the result of selectively attending to spatial locations. Percept Psychophys 28:241-248.

Bisley JW, Goldberg ME (2003) Neuronal activity in the lateral intraparietal area and spatial attention. Science 299:81-86.

Bisley JW, Goldberg ME (2006) Neural correlates of attention and distractibility in the lateral intraparietal area. J Neurophysiol 95:1696-1717.

Brainard DH (1997) The psychophysics toolbox. Spat Vis 10:433-436. 
Cohen MS (1997) Parametric analysis of fMRI data using linear systems methods. Neuroimage 6:93-103.

Corbetta M (1998) Frontoparietal cortical networks for directing attention and the eye to visual locations: identical, independent, or overlapping neural systems? Proc Natl Acad Sci U S A 95:831-838.

Cox RW (1996) AFNI: software for analysis and visualization of functional magnetic resonance neuroimages. Comput Biomed Res 29:162-173.

Dale AM, Fischl B, Sereno MI (1999) Cortical surface-based analysis. I. Segmentation and surface reconstruction. Neuroimage 9:179-194.

Desimone R, Duncan J (1995) Neural mechanisms of selective visual attention. Annu Rev Neurosci 18:193-222.

De Weerd P, Peralta MR 3rd, Desimone R, Ungerleider LG (1999) Loss of attentional stimulus selection after extrastriate cortical lesions in macaques. Nat Neurosci 2:753-758.

Dumoulin SO, Wandell BA (2008) Population receptive field estimates in human visual cortex. Neuroimage 39:647-660.

Egeth HE, Yantis S (1997) Visual attention: control, representation, and time course. Annu Rev Psychol 48:269-297.

Fischl B, Sereno MI, Dale AM (1999) Cortical surface-based analysis. II: Inflation, flattening, and a surface-based coordinate system. Neuroimage 9:195-207.

Friston KJ, Holmes AP, Poline JB, Grasby PJ, Williams SC, Frackowiak RS, Turner R (1995) Analysis of fMRI time-series revisited. Neuroimage 2:45-53.

Greenberg AS, Esterman M, Wilson D, Serences JT, Yantis S (2010) Control of spatial and feature-based attention in frontoparietal cortex. J Neurosci 30:14330-14339.

Grefkes C, Fink GR (2005) The functional organization of the intraparietal sulcus in humans and monkeys. J Anat 207:3-17.

Hagmann P, Jonasson L, Deffieux T, Meuli R, Thiran JP, Wedeen VJ (2006) Fibertract segmentation in position orientation space from high angular resolution diffusion MRI. Neuroimage 32:665-675.

Hagmann P, Cammoun L, Gigandet X, Meuli R, Honey CJ, Wedeen VJ, Sporns O (2008) Mapping the structural core of human cerebral cortex. PLoS Biol 6:e159.

Holmes G (1918) Disturbances of vision by cerebral lesions. Br J Ophthalmol 2:353-384.

Kastner S, Ungerleider LG (2000) Mechanisms of visual attention in the human cortex. Annu Rev Neurosci 23:315-341.

Kastner S, Ungerleider LG (2001) The neural basis of biased competition in human visual cortex. Neuropsychologia 39:1263-1276.

Kastner S, De Weerd P, Desimone R, Ungerleider LG (1998) Mechanisms of directed attention in the human extrastriate cortex as revealed by functional MRI. Science 282:108-111.

Kelley TA, Serences JT, Giesbrecht B, Yantis S (2008) Cortical mechanisms for shifting and holding visuospatial attention. Cereb Cortex 18:114-125.

Lauritzen TZ, D'Esposito M, Heeger DJ, Silver MA (2009) Top-down flow of visual spatial attention signals from parietal to occipital cortex. J Vis 9:18 11-14.

Leber AB (2010) Neural predictors of within-subject fluctuations in attentional control. J Neurosci 30:11458-11465.

Lewis JW, Van Essen DC (2000) Corticocortical connections of visual, sensorimotor, and multimodal processing areas in the parietal lobe of the macaque monkey. J Comp Neurol 428:112-137.

Mars RB, Jbabdi S, Sallet J, O'Reilly JX, Croxson PL, Olivier E, Noonan MP, Bergmann C, Mitchell AS, Baxter MG, Behrens TE, Johansen-Berg H, Tomassini V, Miller KL, Rushworth MF (2011) Diffusion-weighted im- aging tractography-based parcellation of the human parietal cortex and comparison with human and macaque resting-state functional connectivity. J Neurosci 31:4087-4100.

Pelli DG (1997) The VideoToolbox software for visual psychophysics: transforming numbers into movies. Spat Vis 10:437-442.

Posner MI, Snyder CR, Davidson BJ (1980) Attention and the detection of signals. J Exp Psychol 109:160-174.

Potter MC, Levy EI (1969) Recognition memory for a rapid sequence of pictures. J Exp Psychol 81:10-15.

Reeves A, Sperling G (1986) Attention gating in short-term visual memory. Psychol Rev 93:180-206.

Reynolds JH, Chelazzi L, Desimone R (1999) Competitive mechanisms subserve attention in macaque areas V2 and V4. J Neurosci 19:1736-1753.

Saad ZS, Reynolds RC, Argall B, Japee S, Cox RW (2004) SUMA: an interface for surface-based intra- and inter-subject analysis with AFNI. In: Second IEEE International Symposium on Biomedical Imaging: Macro to Nano, pp 1510-1513. Arlington, VA: Institute of Electrical and Electronics Engineers.

Saygin AP, Sereno MI (2008) Retinotopy and attention in human occipital, temporal, parietal, and frontal cortex. Cereb Cortex 18:2158-2168.

Serences JT, Yantis S (2007) Spatially selective representations of voluntary and stimulus-driven attentional priority in human occipital, parietal, and frontal cortex. Cereb Cortex 17:284-293.

Sereno MI, Pitzalis S, Martinez A (2001) Mapping of contralateral space in retinotopic coordinates by a parietal cortical area in humans. Science 294:1350-1354.

Silver MA, Kastner S (2009) Topographic maps in human frontal and parietal cortex. Trends Cogn Sci 13:488-495.

Silver MA, Ress D, Heeger DJ (2005) Topographic maps of visual spatial attention in human parietal cortex. J Neurophysiol 94:1358-1371.

Slotnick SD, Yantis S (2003) Efficient acquisition of human retinotopic maps. Hum Brain Mapp 18:22-29.

Swisher JD, Halko MA, Merabet LB, McMains SA, Somers DC (2007) Visual topography of human intraparietal sulcus. J Neurosci 27:5326-5337.

Talairach J, Tournoux P (1988) Co-planar stereotaxic atlas of the human brain, illustrated edition. Stuttgart, Germany: George Thieme Verlag.

Tootell RB, Hadjikhani N, Hall EK, Marrett S, Vanduffel W, Vaughan JT, Dale AM (1998) The retinotopy of visual spatial attention. Neuron 21:1409-1422.

Uddin LQ, Supekar K, Amin H, Rykhlevskaia E, Nguyen DA, Greicius MD, Menon V (2010) Dissociable connectivity within human angular gyrus and intraparietal sulcus: evidence from functional and structural connectivity. Cereb Cortex 20:2636-2646.

Van Ettinger-Veenstra HM, Huijbers W, Gutteling TP, Vink M, Kenemans JL, Neggers SF (2009) fMRI-guided TMS on cortical eye fields: the frontal but not intraparietal eye fields regulate the coupling between visuospatial attention and eye movements. J Neurophysiol 102:3469-3480.

Verstynen T, Jarbo K, Pathak S, Schneider W (2011) In vivo mapping of microstructural somatotopies in the human corticospinal pathways. J Neurophysiol 105:336-346.

Wandell BA, Dumoulin SO, Brewer AA (2007) Visual field maps in human cortex. Neuron 56:366-383.

Wedeen VJ, Hagmann P, Tseng WY, Reese TG, Weisskoff RM (2005) Mapping complex tissue architecture with diffusion spectrum magnetic resonance imaging. Magn Reson Med 54:1377-1386.

Yeh FC, Wedeen VJ, Tseng WY (2010) Generalized $q$-sampling imaging. IEEE Trans Med Imaging 29:1626-1635. 